\title{
Fitoterapia para el tratamiento de la insuficiencia venosa crónica. El castaño de indias
}

\author{
Phytotherapy for the Treatment of Chronic Venous Insufficiency. \\ The Buckeye
}

\author{
Ma Teresa Sánchez de Membiela \\ Licenciada en Farmacia por la Universidad Complutense de Madrid \\ Máster en Ciencias Farmacéuticas-Fitoterapia por la Universidad Complutense de Madrid \\ tesa_mem@hotmail.com
}

Correspondencia:

Ma Teresa Sánchez de Membiela

Avda de España $41 \mathrm{D}$

E-O2OO2 Albacete

Fecha de recepción: 10 octubre 2010

Fecha de aceptación: 2 febrero 2011

La autora declara no tener ningún tipo de interés económico o comercial.

\section{RESUMEN}

La insuficiencia venosa crónica es una enfermedad común asociada con un gran impacto socioeconómico y a un deterioro significativo de la calidad de vida del paciente.

Actualmente, el tratamiento de referencia son las medias de compresión, sin embargo a menudo causa malestar y una baja adhesividad al tratamiento por lo que se deben tener en cuenta otras alternativas terapéuticas.

Existen diversos fármacos de síntesis para el tratamiento de la insuficiencia venosa crónica. Sin embargo, debido a la cada vez mayor demanda de los pacientes de tratamientos de Fitoterapia y al elevado uso que se viene haciendo en Europa del castaño de indias para el tratamiento de esta patología, se ha considerado relevante ofrecer una pequeña revisión actualizada de los principales aspectos farmacoterapéuticos del extracto de semillas de castaña de indias, cuyo compuesto responsable de la activad farmacológica es la mezcla de saponinas triterpénicas conocida como aescina o escina.

Palabras clave: insuficiencia venosa crónica; Fitoterapia; extracto de semillas de castaña de indias; aescina.

\section{ABSTRACT}

Chronic venous insufficiency is a common disease associated with a large economic impact and a significant deterioration in the quality of life of patients.

Currently, the standard treatment are compression stockings, however this often causes discomfort and a low adhesion to the treatment so other therapies should be considered.

Nowadays, various synthetic drugs for the treatment of chronic venous insufficiency are available. However, due to increasing demand from patients for phythoterapy treatments and the high use that has been done in Europe of horse chestnut for treating this condition, it was considered important to provide a brief review of the main current pharmacotherapeutic aspects of the of horse chestnut seeds extract, which compound responsible for the pharmacological activity is the mixture of triterpene saponins known as aescin or escin.

Key words: Venous Insufficiency; Phytotherapy; Horse Chestnut Seeds Extract; Escin.

Sumario: Introducción. Descripción. Composición química. Propiedades farmacológicas. Farmacología clínica. Indicaciones terapéuticas. Vía de administración, posología y duración del tratamiento. Contraindicaciones. Advertencias especiales. Interacciones. Efectos adversos. Recomendaciones para el paciente. Otras formas farmacéuticas.

Referencia bibliográfica: Sánchez de Membiela, MT. Fitoterapia para el tratamiento de la insuficiencia venosa crónica. El castaño de indias. Rev. Int. Cienc. Podol. 2012; 6(1): 31-37. 


\section{INTRODUCCIÓN}

La insuficiencia venosa crónica es una de las enfermedades más comunes en la práctica clínica .Afecta aproximadamente al 25\% de la población de Europa occidental y es una enfermedad de gran impacto socioeconómico ya que debido a su cronicidad ocasiona largos y costosos tratamientos, que habitualmente requieren hospitalización e intervención quirúrgica provocando un elevado índice de absentismo laboral ${ }^{1}$. Se estima que dichos países dedican entre un 1,5-2\% de su presupuesto sanitario total anual para el tratamiento de la insuficiencia venosa crónica ${ }^{2}$.

Esta enfermedad se caracteriza por un drenaje inadecuado de la sangre venosa e hipertensión venosa. Esto puede deberse a un daño valvular, a un obstrucción venosa o a una combinación de ambos mecanismos ${ }^{3}$. De esta manera se desarrolla una incapacidad de las venas de los miembros inferiores para llevar a cabo el retorno sanguíneo al corazón con la aparición de los siguientes síntomas : dolor y sensación de pesadez, hinchazón, edema, cambios en la coloración de la piel y úlceras que pueden llegar a requerir tratamientos agresivos y costosos llegando a comprometer la calidad de vida del paciente ${ }^{3,4}$.

Entre los factores de riesgo relacionados con esta patología se encuentran: La edad, el sexo femenino, historial familiar de venas varicosas, sedentarismo, obesidad, o antecedentes de trombosis venosa. También se asocian a la insuficiencia venosa factores medioambientales o de comportamiento como situaciones laborales que requieren que el sujeto esté prolongados periodos de tiempo de pie o sentado ${ }^{5,6}$. En las últimas décadas se ha realizado un esfuerzo por parte de los expertos para desarrollar una clasificación clínica bien establecida que siente las bases para un diagnóstico y tratamiento uniforme de la insuficiencia venosa crónica. Así en 1994, el informe de consenso del American Venous Forum presentó la Clasificación CEAP (Clínica, Etiológica, Anatómica y Patofisiológica) cuyos criterios se resumen en la tabla $1^{3,6,7}$. Para mayor información puede consultarse el documento de consenso y sus revisiones ${ }^{8}$.

En cuanto al abordaje terapéutico, el tratamiento de referencia son las medias de compresión, indicadas en todos los estadíos de la enfermedad $^{9,10,11}$. Sin embargo, a menudo causa malestar y baja adhesividad al mismo por lo que se deben tener en cuenta otras alternativas terapéuticas (Bioflavonoides, hidroxirutósidos etc) ${ }^{11,12}$.

De las drogas vegetales más frecuentemente utilizadas para el tratamiento de la insuficiencia venosa crónica cabe destacar el extracto de semillas de castaño de indias que es ampliamente utilizado en Europa y cuyos aspectos principales se describen a continuación:

\section{DESCRIPCIÓN}

El Castaño de Indias (Aesculus hippocastanum L.) es un árbol de hasta $40 \mathrm{~m}$ de altura con hojas compuestas e inflorescencias grandes, su fruto son cápsulas de color verde recubiertas con espículas y dentro de estas cápsulas se encuentran semillas subglobosas, brillantes y pardas que constituyen la droga de esta árbol medicinal. Es originario de Asia Septentrional pero debido a sus llamativas flores se cultiva en todo el mundo como ornamental ${ }^{13}$.

La medicina folklórica describe el uso de

\begin{tabular}{|c|c|c|c|}
\hline CLÍNICA & ETIOLÓGICA & ANATÓMICA & PATOFISIOLÓGICA \\
\hline $\begin{array}{l}\text { Signos clínicos en escala } \\
\text { de } 0 \text { a } 6 \text {. } \\
\text { - Acompañados de A si } \\
\text { son asintomáticos } \\
\text { - S si son sintomáticos. }\end{array}$ & $\begin{array}{l}\text { - Congénita } \\
\text { - Primaria } \\
\text { - Secundaria }\end{array}$ & $\begin{array}{l}\text { Distribución: } \\
\text { - Superficial } \\
\text { - Profunda } \\
\text { - Combinación de } \\
\text { ambas }\end{array}$ & $\begin{array}{l}\text { Causada por: } \\
\text { - Reflujo } \\
\text { - Obstrucción } \\
\text { - Combinación de ambos } \\
\text { mecanismos }\end{array}$ \\
\hline
\end{tabular}

Tabla 1. Resumen de la clasificación CEAP. 
las semillas del castaño de indias para el tratamiento de distintas disenterías bacterianas, fiebres, hipertrofia prostática benigna, alteraciones del aparato circulatorio, respiratorio y urinario entre otros ${ }^{13,14}$. Sin embargo, la única indicación terapéutica aprobada por la Agencia Europea de Medicamentos EMEA como uso bien establecido es el tratamiento por vía oral de la insuficiencia venosa crónica ${ }^{15}$.

En este caso la droga o parte vegetal con actividad terapéutica son las semillas desecadas conteniendo no menos del 3\% de triterpenos glicosilados expresados como Aescina y calculados como referencia a la droga desecada ${ }^{16}$.

\section{COMPOSICIÓN QUÍMICA ${ }^{16}$}

- Saponinas triterpénicas: La mezcla de Saponinas triterpénicas $( \pm 30)$ del castaño de indias recibe el nombre de Aescina o escina, que se pueden fraccionar en $\alpha$-aescina, cryptoaescina y $\beta$-aescina.

- Flavonoides: Di y triglicósidos de quercetina y Kaempferol.

- aninos: proantocianidinas.

- Glúcidos: pricipalmente almidón y oligosacáridos.

\section{PROPIEDADES FARMACOLÓGICAS}

Numerosos ensayos in vitro e in vivo permiten atribuir a la $\beta$-Aescina propiedades venotónicas, antiinflamatorias y antiedematosas. En modelos animales se han puesto de manifiesto las propiedades antiinflamatorias de la aescina mediante un proceso que parece estar relacionado con una disminución de la migración leucocitaria así como una inhibición en la liberación de mediadores inflamatorios. Estos efectos también han sido observados en pacientes humanos tras la administración intravenosa de $5 \mathrm{mg}$ de aescina/ 2 veces al día durante una semana ${ }^{17}$.

La acumulación de leucocitos en los miembros inferiores con la posterior activación y liberación de eslastasa e hialuronidasa constituyen un mecanismo fisiopatológico importante en la insuficiencia venosa crónica (IVC). Se ha demostrado in vitro que la aescina inhibe la actividad de la eslastasa e hialuronidasa, enzimas que participan en la degradación de los proteoglicanos de la matriz extravascular. Al inhibir esta degradación produce una disminución de la permeabilidad vascular lo que favorece la resistencia capilar y disminuye el edema ${ }^{11,16}$.

Así mismo diversos ensayos in vitro e in vivo ponen de manifiesto las propiedades venotónicas de las escina, algunos de ellos se muestran en la tabla 2 y 3 . Todo parece indicar que las propiedades venotónicas se deben a la habilidad de la aescina de aumentar la producción de la $\mathrm{PGF}_{2 \alpha}$ La liberación de este mediador produce una inhibición del catabolismo del tejido venoso y mejora la contractibilidad del mismo ${ }^{17}$.

Por tanto la Agencia Europea del medicamento clasifica el extracto de semillas de castaño de indias dentro del grupo farmacoterapéutico C05: Vasoprotectores y aunque su mecanismo de acción no es exactamente conocido su efecto sobre el tono venoso y la permeabi-

\begin{tabular}{llll}
\hline AUTOR & $\begin{array}{l}\text { MATERIAL } \\
\text { DE EXPERIMIENTACIÓN }\end{array}$ & $\begin{array}{l}\text { EXTRACTO } \\
\text { ADMINISTRADO }\end{array}$ & RESULTADOS \\
$\begin{array}{l}\text { Longiave D. et } \\
\text { al.,1978 }\end{array}$ & Vena Sáfena humana & $5-10 \mathrm{mg} / \mathrm{mL}$ & $\begin{array}{l}\text { Incremento del tono ve- } \\
\text { noso. Se atribuye a un } \\
\text { incremento en la síntesis } \\
\text { de PGF2 } \alpha .\end{array}$ \\
$\begin{array}{l}\text { Guillaume M. } \text { Met } \\
\text { al.,1994 }\end{array}$ & Vena Sáfena canina & $0.0005 \mathrm{mg} / \mathrm{mL}$ & $\begin{array}{l}\text { Contracción máxima } \\
\mathrm{t}=15 \text { min y dura } 5 \mathrm{~h} .\end{array}$ \\
\hline
\end{tabular}

Tabla 2. Ensayos in vitro que ponen de manifiesto las propiedades venotónicas de la Aescina ${ }^{16}$. 


\begin{tabular}{|c|c|c|c|c|}
\hline AUTOR & $\begin{array}{l}\text { ANIMAL } \\
\text { DE EXPERIMENTACIÓN }\end{array}$ & $\begin{array}{l}\text { VÍA } \\
\text { DE ADMINISTRACIÓN }\end{array}$ & DOSIS & RESULTADOS \\
\hline \multirow[t]{3}{*}{$\begin{array}{l}\text { Gillaume M. } \\
\text { et al.,1994 }\end{array}$} & Perros ( vena Femoral) & IV & $50 \mathrm{mg}$ & $\begin{array}{l}\text { Incremento de la } \\
\text { presión venosa. }\end{array}$ \\
\hline & Perros ( vena Safena) & IV & $100 \mathrm{mg}$ & $\begin{array}{l}\text { Efecto comparable } \\
\text { a } 10 \mu \mathrm{g} \text { de NA. } \\
\text { Cierre de válvulas }\end{array}$ \\
\hline & Ratas & V.o & $200 \mathrm{mg} / \mathrm{Kg}$ & $\begin{array}{l}\text { Disminución de } \\
\text { hiperpermeabi- } \\
\text { lidad } \\
\text { cutánea. }\end{array}$ \\
\hline
\end{tabular}

Tabla 3. Ensayos in vivo que ponen de manifiesto las propiedades venotónicas de la Aescina ${ }^{16}$.

lidad vascular parecen estar implicados en sus propiedades farmacológicas ${ }^{15}$.

\section{FARMACOLOGÍA CLÍNICA}

Existen multitud de ensayos clínicos realizados para evaluar la eficacia del extracto de semillas del castaño de indias en la insuficiencia venosa crónica. Bien es cierto que a veces estos ensayos son de poco rigor bien por no incluir un a metodología adecuada o por sólo evaluar un limitado número de pacientes.

En este sentido son de gran importancia las revisiones sistemáticas que permiten combinar los estudios que han intentado responder a la misma pregunta aumentando el tamaño muestral y por tanto incrementando el poder estadístico.

Cabe destacar el trabajo de Pittler M. y Ernst E., $2006^{11}$ que consiste en una revisión sistemática de 29 ensayos aleatorizados controlados con el objetivo de evaluar la eficacia y seguridad del extracto de semillas de castaña de indias por vía oral frente a placebo o frente al tratamiento de referencia para el tratamiento sintomático de la IVC.

En cuanto a los criterios de inclusión de los ensayos fueron los siguientes:

- Los autores sólo seleccionaron estudios que contenían extracto de semillas del castaño de indias como único componente activo (monopreparados).
- Sólo incluyeron estudios en los que los participantes padecían insuficiencia venosa crónica (IVC), seleccionados mediante un criterio de diagnóstico adecuado (p.ej.Widmer, 1978).

- Los autores solo incluyeron ensayos que utilizaran medidas de resultado clínicas (dolor en la pierna, prurito, edema, volumen de la pierna, perímetro de tobillo y pantorrilla y efectos adversos) quedando excluidos aquellos ensayos que se centraban únicamente en parámetros fisiológicos.

De los 29 ensayos controlados aleatorizados identificados inicialmente 12 fueron excluidos por no cumplir los criterios de inclusión de tal manera que quedaron incluidos 17 ensayos.

Todos los ensayos controlados aleatorizados fueron doble ciego excepto uno. De estos 17 ensayos 10 de ellos comparaban el extracto de semillas de castaña de indias frente a placebo; 2 ensayos lo comparan frente al tratamiento de referencia (medias de compresión) y placebo; 4 se controlaron frente al fármaco de referencia (O- $\beta$-hidroxietilrutósido) y uno frente al fármaco picnogenol.

En todos los estudios la administración fue en cápsulas y el extracto utilizado se encontraba estandarizado para aescina. De modo general la dosis diaria consistía en 100-150mg de escina repartido en varias tomas.

Los resultados obtenidos de esta revisión 
sistemática sugieren que en general, el ESCI comparado con placebo y con el tratamiento de referencia es una opción efectiva para el tratamiento de la IVC. Además los efectos adversos observados en los ensayos fueron leves y poco frecuentes por lo que la relación beneficio/riesgo es positiva para el tratamiento de la IVC a corto plazo. Por tanto los autores concluyen que el extracto de semillas de castaño de indias parece ser un opción efectiva y segura para el tratamiento sintomático a corto plazo de la insuficiencia venosa crónica, sin embargo se necesitan más ensayos controlados aleatorizados que deben incluir un gran número de pacientes y evaluar el ESCI especialmente para su uso a largo plazo y como adyuvante en el tratamiento de compresión ${ }^{11,18}$.

Otro autor (Siebert 2002) evalúa 75 ensayos. De éstos 16 fueron incluidos en el metaanálisis (13 ensayos controlados y 3 observacionales). De los 13 ensayos controlados, 11 están concluidos en la revisión de Pittler y Ernst, mientras 2 de ellos aportan nueva información. Los resultados del metanálisis sugieren un beneficio del extracto de semillas de castaño de indias con mejoras significativas en cuanto a la circunferencia de tobillo y pantorrilla, edema y volumen de la pierna ${ }^{18}$.

\section{INDICACIONES TERAPÉUTICAS}

Uso bien establecido. Agencia Europea del Medicamento (EMEA):

Tratamiento sintomático, por vía oral de la insuficiencia venosa crónica caracterizada por hinchazón de las piernas, venas varicosas, sensación de pesadez, dolor, cansancio, picor, tensión y calambres en las pantorrillas.

\section{VIA DE ADMINISTRACIÓN, POSOLOGÍA Y DURACIÓN DEL TRATAMIENTO}

\section{- Adultos y ancianos. Vía oral:}

Extracto estandarizado conteniendo $50 \mathrm{mg}$ de glucósidos triterpénicos calculados como aescina 2 veces al día ${ }^{15}$.

- Duración del tratamiento:

Mínimo 4 semanas para obtener el efecto de- seado. Uso prolongado aceptado bajo supervisión médica ${ }^{15}$.

\section{CONTRAINDICACIONES ${ }^{15}$}

- Hipersensibilidad conocida al principio activo o a las plantas de la familia Hippocastanaceae.

- Embarazo: A pesar de que ESCI se ha utilizado en ensayos clínicos en mujeres en primer y segundo trimestre de embarazo sin la notificación de efectos adversos ${ }^{16}$, estos datos no se consideran suficientes y no se recomienda su uso.

- Lactancia: No existen datos relevantes sobre su uso en madres lactantes por lo que se desaconseja su uso durante la lactancia sin supervisión médica.

- Niños y adolescentes: Debido a la ausencia de estudios y a que no existe ninguna razón terapéutica para su uso en niños no esta recomendado el uso de ESCI por vía oral en menores de 18 años.

\section{ADVERTENCIAS ESPECIALES}

Consultar con el médico en caso de observar:

- Inflamación de la piel, tromboflebitis o induración subcutánea, dolor severo, úlceras y/o hinchazón repentina de las piernas ya que puede deberse a un empeoramiento del trastorno venoso ${ }^{15}$.

- Así mismo en casos de insuficiencia renal, hepática o cardíaca se recomienda extremar las precauciones y consultar con un médico ${ }^{15,19}$.

\section{INTERACCIONES}

Han sido reportados 2 casos de nefropatía probablemente debido a dosis muy elevadas de aescina. Por tanto no se recomienda el uso concomitante de la aescina con otros fármacos nefrotóxicos como la gentamicina ${ }^{14}$.

A pesar de que no han sido reportadas interacciones con otros medicamentos ${ }^{15}$ algunos autores señalan que la aescina podría producir un desplazamiento de la unión a proteínas de 
los anticoagulantes orales, incrementando el riesgo de hemorragia por lo que se recomienda extremar la precaución en estos casos. Así mismo algunos autores señalan que la toxicidad de la aescina podría verse potenciada por antibióticos $\beta$-Lactámicos como las penicilinas y cefalosporinas ${ }^{19}$.

\section{EFECTOS ADVERSOS ${ }^{15}$}

- Molestias gastrointestinales como vómitos, nauseas, hiperácidez y otros cuadros inespecíficos.

- También se han reportado vértigo, dolor de cabeza y reacciones alérgicas.

\section{RECOMENDACIONES PARA EL PACIENTE}

- Aunque la absorción de aescina es mayor en ausencia de alimentos también es mayor el riesgo de daño gástrico por lo que es preferible tomar después de las comidas ${ }^{19}$.

- No superar las dosis diarias recomendadas.

\section{OTRAS FORMAS FARMACÉUTICAS}

Actualmente también pueden encontrase en el mercado formas semi-sólidas estandarizadas en aescina para uso cutáneo. Estas formas están reconocidas por el Committee on Herbal Medicinal Products (HMPC) de la Agencia Europea del Medicamento como producto medicinal tradicional, basándose sus indicaciones exclusivamente en el uso que durante un prolongado periodo de tiempo se ha hecho de las mismas. Estos geles están indicados tanto para el tratamiento sintomático por vía tópica de la pesadez de piernas causada por problemas circulatorios menores o bien para el alivio sintomático de esguinces como edema y hematoma.

En ambos casos la posología para adultos y ancianos consiste en aplicar una fina capa de 1 a 3 veces al día en la zona afectada. No han sido reportadas interacciones con otros medicamentos, el único efecto adverso reportado han sido reacciones de hipersensibilidad en la piel y no está recomendado en mujeres embarazadas ni madres lactantes, tampoco en menores de 18 años (12 años en el caso de los esguinces). Si los síntomas empeoran o aparecen signos de infección en la piel se debe consultar con un profesional de la salud ${ }^{15}$.

\section{BIBLIOGRAFÍA}

1. Ottillinger B, Greeske K. Rational therapy of chronic venous insufficiency-chances and limits of the therapeutic use of horse-chestnut seeds extract. BMC Cardiovascular Disorders 2001; 1:5.

2. Sándor T. Pathomecanism of chronic venous insufficiency and leg ulcer. Acta Physiologica Hungarica 2004; 91 (2):131-145.

3. Eberhardt RT, Raffetto JD. Chronic venous insufficiency. Circulation 2005 May10; 111(18): 2398-409. Review. http://dx.doi.org/10.1161/01.CIR.0000164199.72440.08

4. Koupidis SA, Paraskevas KI, Stathopoulos V, Mikhailidis DP. Impact of lower extremity venous ulcers due to chronic venous insufficiency on quality of life. Open Cardiovasc Med J. 2008; 2: 105-9. http://dx.doi.org/10.2174/1874192400802010105

5. Turchin I, Barankin B. Dermacase. Chronic venous insufficiency. Can Fam Physician. 2006 Oct; 52(10):1217, 1224-5.

6. Fiebig A, Krusche P, Wolf A, Krawczak M, Timm B, Nikolaus S, Frings N,Schreiber S. Heritability of chronic venous disease. Hum Genet. 2010 Jun; 127(6): 669-74. http://dx.doi.org/10.1007/s00439-0100812-9

7. Nicolaides AN; Cardiovascular Disease Educational and Research Trust; European Society of Vascular Surgery; The International Angiology Scientific Activity Congress Organization; International Union of Angiology; Union Internationale de Phlebologie at the Abbaye des Vaux de Cernay. Investigation of chronic venous insufficiency: A consensus statement (France, March 5-9, 1997). Circulation. 2000 Nov 14; 102(20):126-62 .Review

8. Eklöf B, Rutherford RB, Bergan JJ, Carpentier PH, Gloviczki P, Kistner RL, et al. Revision of the CEAP classification for chronic venous disorders: consensus statement. J Vasc Surg. 2004 Dec;40(6):1248-52. Review. 
9. Kecelj Leskovec N, Pavlović MD, Lunder T. A short review of diagnosis and compression therapy of chronic venous insufficiency. Acta Dermatovenerol Alp Panonica Adriat. 2008 Mar;17(1): 17-21. Review.

10. MacKay D. Hemorrhoids and varicose veins: a review of treatment options. Altern Med Rev. 2001 Apr; 6(2): 126-40. Review.

11. Pittler MH, Ernst E. Extracto de semillas de castaña de Indias para la insuficiencia venosa crónica (Revisión Cochrane traducida).En: La Biblioteca Cochrane Plus, 2008 Número 2.Oxford: Update Software Ltd.Disponible en :http://www.update-software.com.( Traducida de The Cochrane Library, 2008 Issue 2.Chichester, UK: John Willey \& Sons, Ltd.).

12. Cheatle TR, Scurr JH, Smith PD. Drug treatment of chronic venous insufficiency and venous ulceration: a review. J R Soc Med. 1991 Jun; 84(6): 354-8. Review.

13. Navarro C, Muñoz Garmendia F. Aesculus hippocastanum L. Flora ibérica Vol 9, http://www.floraiberica.org/[acceso el 22-10-1010].

14. World Health Organization. Semen Hippocastani. WHO Monographs on selected medicinal plants 2004, volume 2: 137-148. Disponible en http://apps.who.int/medicinedocs/en/d/Js4927e/[accesso el 2210-2010].

15. Committee on Herbal Medicinal Products (HMPC).Community Herbal Monograph on Aesculus hippocastanum L., semen. European Medicines Agency. London, 16 July 2009. Disponible en www. ema.europa.eu/ema/pages/includes/document/open_document.jsp?webContentId=WC500059105 [acceso el 22-10-1010].

16. ESCOP Monographs. The Scientific foundation for herbal medicinal products. $2^{\mathrm{a}}$ Edición.Exeter (Reino Unido): ESCOP, Stuttgar: Georg Thieme Verlag, y New York: Thieme New York 2003.

17. No author listed. Aesculus hippocastanum (Horse chesnut). Alternative Medicine Review 2009, 14 (3): $278-282$.

18. Committee on Herbal Medicinal Products (HMPC). Assessment report on Aesculus hippocastanum L.,Semen. European Medicines Agency. London, 16 July 2009. Disponible en www.ema.europa.eu/ ema/pages/includes/document/open_document.jsp?webContentId=WC500059103 [acceso el 22-101010].

19. Consejo General de Colegios Oficiales de Farmacéuticos (CGCOF): Castaño de Indias. Catálogo de plantas medicinales 2010. Madrid. España. 2010:196. 